\title{
Entretien avec Jean-Max Tixier ${ }^{\star x}$
}

\author{
Hédi Bouraoui \\ Université York
}

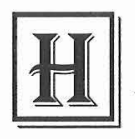

édi Bouraoui : Jean-Max, tu es poète, romancier, essayiste. Tu as pratiqué tous les genres avec succès et bonheur. Et tu $y$ as réussi merveilleusement. Sans être l'ouvre d'un touche-à-tout superficiel, la tienne porte la marque profonde d'une vision éloquente et pertinente. Dis-moi d'abord comment tu es venu à la poésie? Ensuite, pourquoi as-tu privilégié ce genre?

Jean-Max Tixier : Je m’intéresse à la littérature sous toutes ses formes. J'ai voulu m'exercer dans chacune, mû par un défi qui me relance en permanence. Suis-je capable ou non d'écrire tel type d'ouvrage? D'où la grande diversité de mon œuvre. Maintenant riche de près de quatre-vingts titres, elle comprend tous les genres, à l'exception du théâtre. Elle se manifeste à plusieurs niveaux, dans un large éventail qui s'étend du roman de gare sous pseudonyme à la poésie la plus pointue. Depuis mes débuts (première plaquette publiée à dix-huit ans, en 1953), comme l'écrit Michel Cosem, j'ai voulu être "de tous les combats et de toutes les expériences de l'écrit ». Je me définis, non sans provocation, comme un polygraphe. Je fais un pied de nez à qui prétend m'étiqueter et me ranger dans un tiroir, aux censeurs qui désapprouvent la faiblesse de se commettre dans le "populaire " quand on a le talent de créer des œuvres supposées plus dignes. Comme si l'écrivain avait des devoirs envers la critique, le public, la postérité! Je n'accepte pas ce dictat. Je revendique tout ce qui sort de ma plume par respect de la diversité humaine dont chacun est l'exemplaire — l' " humaine condition " chère à Montaigne. Telle est l'expression de ma liberté. Mon être est multiple, tour à tour, gai, triste, grave, fantaisiste, sinistre, hanté par la mort ou optimiste, insouciant, exubérant, mutin, espiègle, farceur,

* Voir notice biographique, p. 52, 73 et poèmes inédits en p. 74. 
pudique ou porté sur la gaudriole. À chaque facette de ce kaléidoscope intellectuel et charnel correspond une place dans le monde des livres. Cette prétendue dispersion m'amuse. Pourquoi m'en priverais-je? Je ne me prends pas au sérieux. Je suis sérieux devant la page blanche.

J'ai tiré de mes pratiques la théorie des stratégies d'écriture. La Littérature, placée une fois pour toutes au sommet de l'Olympe et accessible aux seuls élus, représente un cas particulier de la littérature. Il existe des niveaux littéraires spécifiques. Chacun obéit à ses propres critères de composition, de fonctionnement, de classement, et d'excellence, irréductibles aux autres. J'en distingue trois : littérature de production, littérature de négritude (ghost writter), littérature de création. Cette distribution ne laisse pas d'évoquer la théorie des trois ordres développée par Pascal. Elle a le mérite d'éviter de tout confondre. Ainsi, un roman de poète est-il souvent un roman raté. Cela ne signifie pas que la poésie soit absente du roman mais qu'elle y intervient d'une façon appropriée. Tu as raison de le souligner, il ne s'agit pas des manifestations superficielles d'un touche-à-tout dépourvu d'ambition. La personnalité d'origine garantit l'unité et la cohérence de l'ensemble. Ma vision et ma conception singulières du monde se retrouvent partout, jusqu'au moindre article.

Je réponds maintenant à tes questions sur la poésie. J'ai ressenti très tôt l'émotion poétique et le goût des mots qui lui est étroitement lié. D’abord, grâce à l'école, à la récitation alors en usage. Je me félicite d'avoir encore en mémoire des vers, des poèmes, appris à la communale et au collège. Là me sont venus Hugo, Lamartine, Vigny, mais aussi Baudelaire, Verlaine, Rimbaud. Mon grand-père par alliance, le $\mathrm{D}^{r}$ Gaston Bènes, vieil humaniste agnostique et rabelaisien, m'en a fait découvrir d'autres à domicile : les poètes bohèmes du cabaret Le Chat Noir, les décadents, les fantaisistes, Jean Richepin, Jehan Rictus, Sâr Péladan, mais aussi Paul Valéry, qui me fut précieux avec sa poésie conçue comme " une fête de l'intellect». Gaston me prêtait des livres dont nous discutions après lecture. Par mon grand-père maternel, Max Julien, acteur, auteur de chansons et d'opérettes, me parvenait une poésie plus légère, populaire, facile, un peu fleur bleue quelquefois. Mon amour de la poésie résulte de cette conjonction d'influences. En classe de seconde, un professeur remarquable, M. Lebreton (devenu, par la suite, inspecteur des Lettres) m'a permis de découvrir la Pléiade. Du Bellay reste un de mes poètes de référence, par la rigueur de la composition, la justesse des images, l'exactitude des mots en leur lieu, le respect de la logique poétique. Sont venus à mon adolescence Rimbaud, Mallarmé, Valéry dont la maîtrise me fascinait. J'avais seize ans. 
J'achetais les journaux et les revues littéraires de l'époque dont la plupart ont disparu. J'étais aussi un visiteur assidu de la bibliothèque américaine, située rue Armény, et ouverte au public par le Consulat Général des EtatsUnis, à Marseille. Une poésie différente m’a été révélée. Elle m’a influencé, surtout la lecture de Feuilles d'herbe de Walt Whitman. Je commençais à écrire des poèmes, des paroles de chansons (Marcel Dubel, chef d'orchestre de l'Alcazar, les mettait en musique). J'ambitionnais d'écrire sous l'emprise des grands exemples. Je voulais me rapprocher des grands poètes que j'admirais. Évidemment, j'en étais loin. Je produisais des textes médiocres, sous influence. Évidemment, je n'avais pas posé ma voix.

J'ai eu alors la chance de fréquenter les Cabiers du Sud (1914-1966), une des plus prestigieuses revues internationales, dirigée par Jean Ballard. Au grenier des Cahiers, j'ai rencontré de vrais écrivains, d'authentiques poètes. En particulier, Jean Tortel dont l'exigence m'aida à prendre conscience de la distance qui me séparait de la poésie digne de ce nom, et de tout le chemin à parcourir pour y accéder. Tout en m'encourageant, il refusa de publier mes textes dans les Cahiers du Sud. "Dans votre intérêt », dit-il. Il estimait que je pouvais mieux faire. Je lui dois le conseil le plus précieux que j'aie jamais reçu : "Soyez à l'écoute de votre poème plutôt qu'à l'écoute de vous-même ». Capital! Jusqu'à vingt-sept ans je n'ai plus rien publié. J'ai beaucoup lu, réfléchi, écrit. Ma confidente était la corbeille à papier. Quand j'ai eu le sentiment d'avoir assez progressé pour tenter la publication, je m’y sais risqué. Mon premier manuscrit a été accepté par la revue Encres Vives, qui me décerna son prix annuel, en 1967, pour La Poussée des choses. Je suis entré au comité de rédaction l'année suivante. J'y suis toujours. Je n'ai plus cessé de publier depuis.

La poésie est à mes yeux l'art majeur, la plus haute expression de la littérature, celle qui porte au plus haut degré le travail sur le langage. La poésie est faite avec des mots (cf. Mallarmé). Elle n'a rien à voir avec le spectacle, le déballage public qui prétend toucher par des effets de théâtre. D'où ma réticence envers ce que considère comme de regrettables déviances abusant le public non averti : performances, rap, slam. Ces pratiques sont légitimes, méritent d'avoir leur place, à condition de ne pas tout mélanger et de les considérer pour ce qu'elles sont. Veillons à ce que la société de spectacle ne contamine pas tout par ses facilités. Tu l'auras compris, la poésie représente ce qu'il y a de plus important pour moi. Elle est mon épine dorsale et mon oxygène. Elle me tient droit et me permet de vivre. Mon ami Jacques Lovichi a écrit : "L'écriture / seule / nous tient / debout ». Cela nous réunit. Je le prends à mon compte. 
H. B. : Poésie profonde, parfois elliptique, parfois lyrique, toujours d'une percutante originalité. J'avoue que jaime beaucoup ton écriture poétique. Simplement parce qu'elle me parle. Elle minterpelle et jy adhère tandis qu'elle me permet de me remettre en question. Peux-tu évoquer, pour la revue LittéRéalité, ton faire poétique, autrement dit, ta Poïetique?

J.-M. T. : On ne décide pas d'une poétique. Elle s'impose au fur et à mesure du travail, de la réflexion qui l'accompagne, des problèmes internes qui se posent et des solutions pratiques que l'auteur est ou non capable d'apporter. L'interrogation sur la poésie est au cœur du poème. Sartre dit quelque part : "Il faut penser contre soi-même ». J'adapterais la formule en ces termes : il faut écrire contre soi-même, c'est-à-dire se dégager des apparences, de la complaisance, de l'image flatteuse qu'on veut donner de soi, rompre avec son moi familier et social pour atteindre, dans les profondeurs de l'être, le moi poétique, seul digne d'intérêt. Il s'agit d'une hygiène des lettres qui met en jeu une éthique. Je suis matérialiste, incroyant, méfiant envers la subjectivité, la spontanéité, étranger à la métaphysique, indifférent à la transcendance, imperméable au sacré.

Pendant des années, j'ai lutté contre le lyrisme, dont la force me subjuguait, et qui se manifestait à mes débuts. Je m’en suis affranchi non sans difficulté. J'ai opéré, à travers de pénibles épreuves, des ruptures et des arrachements successifs, pour passer du lyrisme de surface à ce que j'appelle le lyrisme des profondeurs - Baudelaire parle déjà de " rhétorique profonde ", et on retrouve cela chez Saint-John Perse. Le lyrisme des profondeurs échappe aux facilités et aux séductions de la dominante rythmique dont le danger est de détourner de l'essentiel au profit d'une exaltation délectable. Ma démarche poétique s'inscrit et se développe dans cet espace. Je m'efforce de faire en sorte qu'elle échappe aux sollicitations extérieures pour obéir à sa propre logique. Ne nous méprenons pas : ceci n'a rien à voir avec le vieux débat de la poésie pure. Il s'agit d'une conception objective, dynamique, quasi scientifique, en prise directe sur le monde historiquement posé, ou, pour reprendre un de mes titres, l'état des lieux. Pour être vraiment contemporaine, la poésie ne doit pas se transformer sous le seul effet des forces en jeu dans le fait poétique. Elle doit prendre en compte et intégrer dans son champ celles des sciences qui façonnent et éclairent le moment, en subordonnant les sciences dites humaines à celles de la matière.

Ma poïétique ne prend pas appui sur un corps de doctrine ni ne se réfere aux dictats d'une école. Son apport théorique est inclus dans son fonctionnement même qui, sans cesse, la prend en défaut et la relance vers 
de nouveaux apports provisoires. Chaque poème contient sa propre critique. Il révèle des manques, des failles, des inadéquations, des contradictions, qu'une étape ultérieure prétendra résoudre conformément à leur nature, sans résultat définitif. Le renouvellement est à ce prix, dans le bon usage de l'insatisfaction. Mon travail de poète s'articule à deux axes principaux : le poème est instrument de connaissance objective et relative; le poème rénove en permanence le langage. Il établit de nouveaux rapports au monde rendus nécessaires par les agents de transformations en mouvement. S'impose la nécessité de se réadapter sans cesse au nom de tous, de restituer aux hommes le monde dont ils se croient dépossédés. Robert Sabatier voit en moi un «laveur de mots». L'expression me plaît. Le poète lutte contre l'usure des mots, la déperdition de leur valeur et de leur énergie. Rien n'est plus opposé aux lois qui régissent la mondialisation capitaliste.

H. B. : Ton processus poétique infuse au lecteur éventuel une mystérieuse boulimie de lecture qui renouvelle le sens des Etres et des Choses. Quels sont pour toi les ingrédients essentiels qui accrochent les lecteurs de poésie?

J.-M. T. : La réponse à cette question complétera celle de la précédente. Je refuse de parler d'ingrédients parce que cela accréditerait l'idée de recettes, de cuisine littéraire. Quand j'écris, je ne pense pas aux lecteurs, à la façon dont ils accueilleront et jugeront mon texte. C'est hors de question. L'esprit se concentre sur les problèmes poétiques à déceler et à résoudre. Toutes les ressources de l'auteur sont mobilisées en vue de cet objectif. Le souci du lecteur intervient après, quand le travail, supposé achevé, va être rendu public. Si le processus poétique produit les effets que tu soulignes, c'est en raison de l'importance de l'investissement du poète dans la création poétique. Le poème, dans sa forme finale, est un lieu de forte densité, de concentration maximale, une structure harmonique qui trouve sa cohérence et sa dynamique interne à partir d'un grand nombre de variables. Le faire esthétique conduit à l'objet esthétique, mais pas seulement. Fruit de la lucidité, du hasard, du savoir, de la ténacité, il doit d'être ce qu'il est à toutes les ressources affectives, intellectuelles, sensuelles du poète. Dans ces conditions, il sollicite, plus que tout autre type de texte au tissu plus lâche, une lecture participative, active, créatrice. Les concepts d'émergence et de téléonomie, empruntés à la biologie moléculaire, sont applicables ici. J'ai beaucoup appris de l'ouvrage de Monod Le Hasard et la nécessité.

Lorsque je lis certains poètes majeurs, un moment vient où ils agissent sur moi avec tant de force irradiante, communicative, que je suis contraint 
d'abandonner ma lecture pour réfléchir ou convertir l'impulsion reçue en écriture. Le poème est le tremplin du poème. Ce feu soudain qui prend aux mots constitue l'ardeur poétique - la fureur divine des anciens. Les propositions inédites déchirent le voile recouvrant les choses, dégageant brusquement un espace nouveau donnant carrière aux aventures de l'esprit, l'occasion d'une autre naissance. D'où cette boulimie, cette excitation dont tu parles à propos. Je la ressens moi-même en lisant certains poètes. J'ai coutume de dire que ce sont des œuvres habitables.

H. B. : On dit souvent que la poésie ne se vend pas. Ses lecteurs sont rares. Les éditeurs ne veulent plus investir dans ce genre. Et pourtant, en France, il y a plus de soixante milles poètes, presque un poète pour mille habitants. Puisque tu es un poète prolifique et un poète majeur de la scène poétique française, dismoi comment tu réagis à ces constatations.

J.-M. T. : Nous sommes en présence d'un paradoxe qui nous interpelle. D'une part, le nombre de poètes n'a pas diminué, au contraire. De grandes voix contemporaines s'imposent par leur diversité et leur originalité. De sorte qu'on peut souligner avec raison la vitalité de la poésie d'aujourd'hui. Elle n'a rien à envier à celles qui l'ont précédée. On en parle cependant moins parce qu'elle est constituée d'individus occupés à tracer leur propre voie dans la discrétion. Les phénomènes d'écoles, de groupes, de coteries, les conflits théoriques, les guerres fratricides entre tendances opposées, ont quasiment disparu. Ils n'occupent plus le devant de la scène. Ils ne produisent plus d'événements capables de mobiliser l'attention des médias à l'affût du sensationnel. La poésie a-t-elle besoin de cela pour être? Non, mais l'histoire de la poésie oui. Nous vivons dans une société de consommation qui tend à réduire toutes les manifestations du génie humain à l'état de produits soumis à la loi du marché. La valeur se mesure à la quantité écoulée, au retentissement. Or, l'une des fonctions de la poésie est justement de résister à la marchandisation à laquelle elle est par nature irréductible. Un recueil vendu à cinq exemplaires peut être plus important que celui vendu à trois mille. La valeur est souvent inversement proportionnelle au tirage. Il est préférable d'être lu par dix ou vingt lecteurs compétents que par dix mille incapables de comprendre. Élitisme? Pourquoi pas? Je ne recule pas devant le mot, à condition d'en bien saisir le sens.

Il existe un public potentiel plus large qu'on ne le prétend. Cependant, rien n'est mis en œuvre pour le gagner et le fidéliser. Le circuit de diffusiondistribution d'un recueil est identique à celui d'un roman, ce qui le 
condamne d'emblée. La faiblesse du tirage ne permet pas une mise en place correcte dans les points de vente. Les libraires, croulant sous les offices, renvoient les livres au bout de six semaines alors que la poésie exige une rotation lente de plusieurs mois, voire plusieurs années. Les éditeurs de poésie sont de petites maisons indépendantes négociant un faible volume d'exemplaires. Ils n'intéressent donc pas les distributeurs. Pour ne pas perdre un temps précieux à chercher dans le stock, ceux-ci déclarent quelquefois épuisés des livres qui ne le sont pas. Le système n'est donc pas conçu pour servir la poésie. Il serait injuste d'incriminer de tous les maux des auxiliaires du circuit qui ne sont que des commerçants préoccupés par leur chiffre d'affaires. Il faut stigmatiser la responsabilité des poètes de tous poils et des amateurs de poésie. Il existerait plus de soixante mille poètes déclarés en France. J'accepte l'évaluation. Parmi ceux-ci combien y en a-til qui lisent d'autres poésies que la leur? Combien achètent un ou plusieurs recueils par an? Je reçois souvent de prétendus poètes qui ambitionnent d'être publiés. Ils me présentent leurs manuscrits. Chaque fois, je pose les mêmes questions : quel est votre poète préféré? Quels sont les poètes contemporains que vous connaissez? Quel est le dernier recueil que vous avez lu? Huit fois sur dix, ils ne répondent rien parce qu'ils ignorent tout. Par quelle aberration déplorerait-on la faiblesse des ventes de la poésie quand les poètes eux-mêmes ne lisent pas les poètes?

Imaginons de nouvelles procédures de diffusion sans pour autant rompre avec les circuits commerciaux habituels au nom de la liberté et de la pluralité. Il n'est pas souhaitable, par exemple, que la loi du marché ayant évincé les petits éditeurs indépendants, les Universités y suppléent en se chargeant de l'édition poétique. J'estime cette collusion malsaine, fût-elle imposée par la force des choses. Rien n’est plus éloigné de la poésie que le discours universitaire. L'une est contestation, franchissement, entreprise de liberté, bref, création, l'autre intervient une fois l'œuvre accomplie pour décrire, classer, analyser, commenter, établir des grilles d'interprétation, théoriser. En ce qui me concerne, de 1967 à aujourd'hui, je ne me suis pas heurté à des obstacles insurmontables pour trouver des éditeurs et bénéficier, avec le temps, d'une relative audience. L'obtention de quelques prix m'a aidé, certes, mais il faut souligner le peu d'effet d'un prix sur les ventes. Plus utile s'est révélée mon appartenance aux comités de rédaction de plusieurs revues: Encres Vives, Sud, Impressions du Sud, Autre Sud, Poésie 1/Vagabondages, et ma collaboration, plus ou moins fréquente, à d'autres. Je crois au rôle des revues de poésie, même si elles ont perdu de leur influence au cours des dernières décennies. 
H. B. : On reproche aux poètes à succès leur hermétisme. Puis-je soulever avec toi les problèmes $d u$ " trobar clus " et du "trobar clar "? Je sais plus ou moins où tu te situes par rapport à ces deux tendances de la poésie française. Peux-tu expliciter ta tendance et tes penchants?

J.-M. T. : Le problème de l'hermétisme est fondamental. Il justifie le reproche d'illisibilité encouru par la poésie contemporaine. Essayons d'y voir plus clair. Le mot recouvre des réalités différentes, voire opposées. Rechercher l'obscurité pour elle-même, l'exploiter afin de masquer son impuissance créatrice, cultiver l'inaccessible pour prendre posture, ceci est inacceptable en tant que pose et supercherie. On n'est pas profond parce qu'on est obscur. Au contraire. Il faut dénoncer aussi les épigones, imitateurs maladroits du mâttre, qui, malgré leur admiration, ne sont pas parvenus au cœur de son énergie créatrice. N'ayant pas assimilé sa démarche, ils s'en tirent avec des trucs, des emprunts, des détournements, dans le vain espoir de faire illusion. Ainsi de certains suiveurs de Mallarmé. L'hermétisme peut également provenir de l'ésotérisme, du goût ou de l'obligation du secret, du symbolisme réservé aux initiés. Dans ce cas, ce qui est hermétique pour les uns - la masse - ne l'est pas pour les autres. D'une façon générale, ce caractère n'est ni absolu ni définitif. L’hermétisme ou, si l'on préfere, la difficulté d'accès d'un texte, résulte de la différence de niveau entre le poète et son lecteur, à un moment donné. Le poète, en décalage, en avance sur son époque, use d'un langage précurseur. Peu de gens comprenaient Rimbaud à la publication des Illuminations. Un siècle plus tard, il est étudié au lycée. Cet hermétisme-là est inséparable de l'évolution de la poésie. Il est juste et inévitable. C'est un hermétisme de fonction. Un poète écrit en assumant l'histoire de la poésie à laquelle il est redevable de ce qu'il est et de ce qu'il fait. Son arbre généalogique est constitué de tous ses prédécesseurs - parmi lesquels quelques branches putatives. Il est porté au-devant de ces générations, au seuil où, d'un langage nouveau, il les dépassera. Il faut s'appuyer sur le même socle de culture pour le comprendre. Or, le grand public l'aborde à partir d'une culture traditionnelle, insuffisante et morcelée. On ne passe pas avec aisance de La Fontaine à Francis Ponge. Le différentiel ne peut être, dans tous les cas, résorbé dans l'immédiat. Le travail poétique, dans la mesure où il ne transige pas et où il n'est pas dévoyé de son cours, conduit inévitablement à l'hermétisme, mais un hermétisme légitime et cohérent - en poésie comme en science le principal critère est, comme l'avait compris Roger Caillois, la cohérence. Prenons un exemple pour mieux le faire entendre. Quand un mathématicien trouve un nouveau théorème

\section{LittéRéalité}


combien de ses pairs s'avèrent-ils capables de le comprendre immédiatement? Quand Einstein établit sa théorie de la relativité, corroborée par l'observation et l'expérience, la majorité des physiciens s'avouent dépassés par ses conceptions. Il en va de même d'un langage et d'un univers poétiques résolument novateurs. Paradoxalement, c'est en allant vers plus de clarté et de synthèse simplificatrice qu'on engendre ce sentiment d'hermétisme. Je me situe dans ce dernier courant, sans m'abuser et en m'entourant de toutes les précautions utiles. Pas d'obscurité artificielle et inutile, mais une recherche constante à la pointe de l'inquiétude poétique aux prises avec le réel. Sur cette ligne, tout se passe comme si, avec le temps, le "trobar clus" devenait le " trobar clar ". Ma poésie étant résolument tournée vers la connaissance plutôt que vers l'expression, je m’intéresse depuis longtemps aux rapports entre la poésie contemporaine et les sciences. J'ai soutenu une thèse sur ce sujet. Il ne s'agit certes pas d'assimiler la poésie à une science, encore moins de la mettre en équation, mais de mieux comprendre et exploiter les analogies de fonctionnement intellectuel dans deux domaines réputés opposés. Du fait que l'esprit s'applique à deux supports différents, on ne saurait inférer que les aptitudes mobilisées soient également différentes. Nous recourons aux mêmes outils intellectuels en les adaptant chaque fois à la nature des choses.

H. B. : Si je remonte à ta poésie lointaine, en amont de ton itinéraire, je constate que ton recueil La Pierre hypnotisée inaugure en quelque sorte ton style métaphorique. D'ailleurs, une grande partie de ton ceuvre est constituée de diverses cascades de métaphores. Je te rappelle en passant un quatrain de ce recueil : "Laisse la gloire taillée par trop de mains / Ta vérité est une métaphore / Ta seule arme le chant / Et ta patrie la vie ». Il me semble que nous avons là ton art poétique. Peux-tu commenter pour nous les fonctions de la métaphore et de chant?

J.-M. T. : Je me méfie désormais du chant en poésie. J'ai affirmé plus haut mon refus du lyrisme et dit comment je m'étais efforcé de lui tordre le cou, aidé en cela par le travail collectif effectué au sein du groupe Encres Vives, au cours des années soixante-dix. Nous établissions une rupture radicale avec les productions poétiques de l'époque en vue de parvenir à une écriture nouvelle, matérialiste et objective, privilégiant le travail sur la langue. Nous nous étions approprié deux phrases citations en forme de slogans pour situer notre démarche, l'une de Julia Kristeva : "Faire de la langue un travail ", l'autre de Roland Barthes : "Le sens aboli tout reste à faire ». Nos recherches respectives, mises en commun, objets permanents 
de critiques collectives, où l'auteur en tant que tel perdait ses prérogatives, se fondaient sur la réflexion théorique articulée à la pratique et sur l'expérimentation. De sorte qu'on nous a classés parmi les représentants de l'écriture textuelle, avec les gens de la mouvance de Tel Quel et de TXT. Ce n'était que très partiellement vrai. Notre revue, quoique de moindre notoriété, était jalouse de son indépendance et suivait des orientations originales (sur le mythe, la rupture de la linéarité, la reprise). Le recueil auquel tu te réferes, La Pierre hypnotisée, date de 1968, donc du début de ce processus dont il n'est pas représentatif, même s'il l'annonce par des indices incontestables. La subjectivité, l'effervescence communicative de l'émotion, l'envolée rythmique, voire une certaine forme d'engagement, y représentent encore une part importante. À l'époque, je ressentais les choses ainsi et j'étais sincère. J'ignorais encore que la sincérité n'est pas un critère d'appréciation de l'art. Les vers cités expriment mon art poétique du moment. Sans doute en reste-t-il quelque chose, une fidélité aux ferments de la jeunesse qui m'ont fait ce que je suis aujourd'hui. J'utilisais le mot chant dans son acception première, sans voir qu'il ne s'agissait aucunement de chanter, le verbe porté par la musique, soulevé par la vague rythmique vers un au-delà du langage, soumis à l'hypnose des sons, mais de célébrer l'ordre des choses, de le transformer esthétiquement pour en extraire la puissance latente, les rapports sous-jacents reliant la matière. Or, il ne faut pas que cet agrément donne le change sur la profondeur de la pensée, ni qu'on oublie à son profit la densité signifiante de ce réseau de relations. La seule approche possible du poème consiste dans la lecture silencieuse qui confronte le lecteur à l'espace de la page où figure la géométrie sensible du poème, ajustement de signes dans un ordre immuable. Le poème est construit avec des mots, phonétiquement distincts, harmonieusement organisés selon des critères spacio-temporels. Il a, de ce fait, une tessiture musicale. Mais, d'une part, elle n'est pas première, d'autre part, elle résulte de la constitution même des vocables, de l'exactitude de leur choix, de la justesse de leur place dans l'ensemble verbal. La musique est une propriété des mots parmi d'autres, de sorte qu'elle doit être sensible dans une lecture blanche évitant les effets interprétatifs de la déclamation. Je me situe à l'opposé de Verlaine prescrivant " de la musique avant toute chose ».

J'aime la figure de la métaphore, source inépuisable d'images, tout en me gardant de sa séduction et des dangers qu'elle présente. Je relève souvent, dans certains recueils, des amas de métaphores incohérentes ou incongrues fatales au poème. Elles ont été retenues pour leur puissance, leur singularité, sans prise en compte de leur fonction dans l'ensemble. Or, l'emploi de la métaphore exige circonspection et rigueur. Dans un poème 
de la suite intitulée Profils de chutes et Autres partitions (2003), j'ai écrit : "La métaphore verse ses eaux grasses à plein seaux". J'ai souvent abordé ces questions, au sein de la revue Sud, avec mon ami Pierre Caminade, auteur d'un essai pertinent: Images et métaphores. Je ne recherche pas la métaphore pour elle-même. Quand je peux l'éviter, je le fais. De même, des images en général. Trop d'images tue l'image. Je ne partage absolument pas la fascination des surréalistes pour "le stupéfiant image ". Ma poésie ne trace pas sa voie hors de la maîtrise et de la conscience lucide de son fait. Si je lâche parfois la ligne, c'est intentionnellement, pour produire un effet particulier, et la reprendre aussitôt. Je me sens plus proche de Roger Caillois que d'André Breton et de ses acolytes. Je pense n'avoir pas subi l'influence des surréalistes, sinon dans mon âge tendre. Je n'entends pas perdre prise sur le monde au profit d'un état mental altéré, fût-il agent de plaisir. Je préfere tenter de me trouver à la faveur du poème plutôt que de me détruire par l'abus des drogues verbales. L'image, la métaphore, telles que je les conçois et en use, favorisent l'accès à la connaissance. Je m'applique à poser les mots au plus juste d'une tension. Ce sont ces lignes de force qui importent. Je tâche de les rendre sensibles, sinon visibles. À ce moment-là, elles deviennent opératoires. Pour ce que j'en sais, cela paraît comparable à ce qui se produit dans certaines disciplines scientifiques, par exemple la physique ou la biologie moléculaire. L'emploi de la métaphore doit respecter la logique poétique.

H. B. : A lire les titres de tes livres de poésie, je remarque quills tournent souvent autour de la question du temps : L'Arrière-temps, L'Instant précaire, Où s'invente le jour, Le Temps des guêpes, du lieu : Lecture d'une ville, La Traversée des eaux, États des lieux, et des éléments, terre, eau, feu, air: Le Jardin d'argile, L'Oiseau de glaise. Comment choisis-tu tes titres et quelles fonctions leur donnes-tu?

J.-M. T. : Le temps et le lieu constituent les deux pôles de ma poésie. Il faut affiner car ces notions recouvrent plusieurs choses. Pour la première, la relation entre le temps du poème et le temps chronologique qui, opérant un découpage significatif de l'espace en mouvement, produit une réalité opératoire qui règle les actions humaines; la relation entre ce système et sa traduction verbale dès lors qu'il entre dans la conscience humaine et traduit la durée, sensation relative de son écoulement. Le surgissement du temps marque la rupture de l'unité originelle. Il inscrit une fracture, une blessure ontologique que la poésie tente de résorber. Dans Hier régnant désert, Yves Bonnefoy écrit : " Enfièvre encore en toi le temps qui va guérir ». Je pense 
que la poésie, comme les mathématiques, peut opérer sans le temps — d'où la fréquence du présent qui, comme le démontrait Gustave Guillaume, est lui-même un temps dégagé de l'emprise du temps, projeté hors du vecteur d'écoulement de celui-ci, point cursif, sans épaisseur, sans passé ni futur. L'énergie poétique se libère dans ce changement d'axe, ce que je nomme L'Instant précaire (1995). Le poème, en s'affranchissant du discours, de ce qui est dit dans le décours du temps, échappe à sa coulée. Je travaille dans ce complexe selon mes besoins. Ainsi, l'emploi d'un temps verbal trouve-til quelquefois sa place non pour exprimer ce pour quoi il est fait grammaticalement, mais, d'un point de vue structurel, pour établir un contraste, fixer dans le texte un télescopage verbal, pour sa valeur vocalique, ou tout autre chose. Par ailleurs, le temps est ce qui est enfermé, fixé, dans la mémoire, une sorte de temps archéologique, ethnologique. Tel est le sens de mon recueil Chasseur de mémoire (2001).

La question du lieu s'avère également complexe. Elle est fondamentale en poésie contemporaine, par exemple chez un poète comme Yves Bonnefoy. Il ne suffit pas de dire d'où l'on parle, encore faut-il savoir où se situe le poème. En jouant sur les significations, je dirais que la poésie doit avoir lieu, se manifester en étant situable. Lors de mes recherches en vue de ma thèse, j'ai émis l'hypothèse d'un lieu poétique analogue au lieu géométrique. Il désignerait celui où l'ensemble des éléments constitutifs justifieraient d'une propriété exclusivement poétique. Soit encore, l'espace mental défini par la conjonction des forces qui concourent à l'inscription et au rayonnement du poème. Le lieu de la poésie, c'est la langue, et nulle part ailleurs, sauf à confondre poésie et sentiment poétique. "Pas de patrie ni de demeure ailleurs que dans la langue en tant que poésie ", écrit Adonis. Je ne prends pas le terme dans son acception géographique qui inviterait à la description, au commentaire, voire à une quelconque célébration plus ou moins affective et réaliste. Quand j'écris États des lieux (1992), je ne m'interroge pas sur autre chose que la situation du poème dans mon propre itinéraire. Je circonscris une aire à l'intérieur de son domaine spécifique. Le poème prend à l'endroit où se développe la germination du langage. Là se déroule le travail de création. Dans une suite intitulée justement Saisir le lieu, et dédiée à Jean Digot, je mets en avant le caractère géologique et anthropologique de cette démarche : « Il te faudra longtemps fouiller la profondeur des strates avant d'atteindre en son lieu l'écriture qui t'ouvrira la mer ». On retrouve cela dans mon avant-dernier recueil, Chasseur de mémoire (2001).

Cette citation me permet d'aborder la dernière partie de ta question relative aux éléments. Nous connaissons leur pouvoir symbolique depuis 
les travaux de Gaston Bachelard et leur fonction essentielle dans la création poétique. Je n’y échappe pas. Mes métaphores empruntent selon les besoins à la terre, l'eau, l'air, le feu. Il faut cependant tenir compte de la dominante et de la fréquence. J'ai toujours privilégié la terre, le minéral, parce que cela correspond à ma sensibilité et à ma poétique. Toute trace équivaut à un signe, provoque la lecture, l'interprétion. Tout se passe comme si nous étions environnés d'une profusion d'alphabets appartenant des idiomes inconnus et chargés de mystère, un monde constitué d'énigmes à élucider. Le travail du poète - enfin, le mien - consiste à doter de significations ce qui n'en a pas, c'est-à-dire les failles, les fissures, les fractures, les nodosités, les creux, le lisse et le rugueux, à interpréter les architectures naturelles ou humaines - d'où mon recueil Lecture d'une ville (1976). Je cherche à comprendre l'écriture des pierres, La pierre écrite d'Yves Bonnefoy. Je me sens ainsi dans la proximité de poètes comme Roger Caillois et Lorand Gaspar.

Le titre n'est pas une simple étiquette. Il fait partie du poème et désigne le champ poétique dans lequel il se situe. Il fait quelquefois office d'embrayeur lorsqu'il est trouvé le premier, d'un stimulateur de création. En même temps, il donne une clé du recueil. Ses fonctions sont donc multiples. Le choix du titre est d'une grande importance. C'est lui qui accroche ou non le lecteur en communiquant un ton, une thématique, un espace. Il en va de même pour le titre d'un chapitre, d'une suite, ou d'un poème - en général, je ne donne pas de titre aux pièces indépendantes. Encore qu'on puisse considérer les fragments d'une suite comme les strophes d'un même poème.

H. B. : Il est bien évident qu'on ne peut synthétiser les thématiques d'un auteur en ayant recours au raccourci des titres! Cependant, je crois que pour toi, comme pour Mallarmé, la poésie est avant tout affaire de mots. Leurs accords, leurs rivalités, leurs contrastes et leurs contradictions. C'est eux qui créent un "climat » poétique, pour reprendre l'un de tes titres, Questions de climat. Peux-tu te positionner par rapport à ce constat?

J.-M. T. : Le titre n'est pas innocent. Il participe du poème. Il en pose les premiers mots. J'adhère sans réserve à la conception mallarméenne qui fait de la poésie une affaire de mots, et aux phases de son évolution jusqu'à Ponge, qui prescrit le " compte tenu des mots ", et au delà. Le mot représente la cellule de base qui permet toutes les associations - malgré les tentatives des lettristes -, comme un élément chimique avec ses valences, ses variations quantiques, mais infiniment plus complexe. Je l'ai défini 
comme un plurilogisme. Le dispositif d'ensemble par les qualités des éléments constitutifs, leur place dans le dispositif, leur enchaînement, gouverne l'état mental produit par la lecture - la perception. D'où la sensation d'un climat verbal. Frédéric Jacques Temple parle à ce propos d'instants « nés de l'accord ou de la rivalité des mots".

H. B. : Plusieurs de tes recueils sont en prose poétique. Est-ce pour te démarquer de la métrique traditionnelle? De la rime ronronnante? Du discours déclamatoire? Explique-nous un peu tes raisons concernant cette option.

J.-M. T. : Je n'ai pas choisi d'écrire des poèmes en prose. Le poème en prose s'est imposé à moi parce que, à un moment donné, il correspondait à une nécessité. Depuis, j'affectionne particulièrement le genre auquel j'ai beaucoup réfléchi. À partir de petites unités, il montre comment la poésie investit la prose et pourquoi il advient que celle-ci contienne plus de force poétique que certains poèmes. S’il suffisait d'aller à la ligne pour l'apprivoiser, de régler une coupe, de poser une rime! L'usage démontre le contraire. La fortune du poème en prose, depuis Aloysius Bertrand, en passant par Baudelaire, Rimbaud, Lautréamont, plus tard Saint-John Perse, Char, Ponge, révèle quels liens étroits il entretient avec la modernité. Certaines choses ne peuvent être dites que par le poème en prose. C'est lorsque j'ai éprouvé l'insuffisance, l'inadéquation plutôt, du poème versifié à ce que j’entendais manifester, que je me suis tourné vers lui. Il m'apportait les solutions attendues. Je me contenterai de quelques aperçus. Chez moi, le poème en prose, constitué le plus souvent d'un seul paragraphe, relève de l'architecture. Il pose sur la page un bloc compact, géométrique, de matière, en opposition avec le blanc environnant. Les éléments constitutifs, taillés au plus juste, sont joints comme les pierres sèches d'un mur qui tiennent par l'équilibre de l'ensemble, et, jouant les unes par rapport aux autres, produisent un effet esthétique. Cet aspect convient particulièrement à ma technique de la double ponctuation qui ne tient pas compte des règles de la grammaire. Un seul mot - substantif, verbe - ou une expression peuvent être placés entre deux points, par exemple, pour être valorisés, mis en relief dans le contexte, faire que tout ce qui figure entre deux points équivaut à un mot tout en conservant sa signification première. Ce procédé permet de casser le lyrisme par des ruptures syntaxiques. Il permet aussi d'introduire dans le texte des lieux d'incertitude, des variations de sens : le fragment placé entre deux points s'accorde également avec ce qui précède et ce qui suit. La continuité de la 
prose rassemble, en un lieu mieux circonscrit, les réseaux de relations constitutifs du poème. Elle défend celui-ci d'interprétations étrangères. Enfin, dans un souci stratégique d'efficacité, par la préparation psychologique qu'on peut mettre en place pour cette seule fonction, il confere le maximum d'intensité aux aphorismes poétiques. Insister m'entraînerait trop loin. Il va de soi que je rejette catégoriquement métrique traditionnelle, rime ronronnante, discours déclamatoire. Lorsque je constate que certains poètes d'aujourd'hui tentent, pour des mobiles qui m'échappent, de les restaurer, j'en suis profondément irrité. On ne peut entrer sans dommage dans la peau d'un Grand Rhétoriqueur, d'un représentant de la Pléiade, d'un petit maître du XvıII , où d'un lyrique romantique. Il s'agit soit d'une orientation artificielle, plus ou moins justifiée par le goût des contraintes, sous l'influence perverse de l'Oulipo, soit d'un refuge contre la contemporanéité, soit d'un aveu d'impuissance. La poésie de notre siècle doit être capable de progresser sans béquilles.

H. B. : Souvent, chez toi, les mots deviennent peinture, musique, lumière. Appel à d'autres arts? Incursion inter-artistiques? Traitement de la poésie sous toutes les formes d'art? Qu'en penses-tu?

J.-M. T. : Il existe des relations, plus ou moins étroites et fécondes, entre les arts. Moi, j'ai toujours été particulièrement sensible aux arts plastiques - j'ai écrit sur les peintres, les photographes. On connaît depuis Baudelaire la proximité de la peinture et de la poésie. J'ai dit combien m’intéressait l'architecture. Dans ma jeunesse, je lisais des ouvrages sur Wright, le Bauhaus, Le Corbusier, l'urbanisme. J'ai dit plus haut que ma poétique se fondait sur l'organisation du minéral, l'interrogation des traces. Or, un tableau, figuratif ou abstrait, présente une structuration de l'espace, l'expression d'un langage formel directement lié à la perception spécifique de l'artiste. Il s'en dégage un champ poétique dans lequel un poète peut se mouvoir, risquer un itinéraire au milieu de cette conjonction d'images, de taches de couleurs, de représentations interprétatives du réel. Regarder un tableau stimule mon imaginaire, fait naître le besoin de créer. J'ai publié certaines suites qui n'existeraient pas sans de telles rencontres. Ainsi, Ouverture du delta (1980) est né de ma lecture de photographies de mon ami Lucien Clergue présentées sous le titre Camargue secrète. Il faut considérer que la forme s'offre dans une profusion d'analogies, de correspondances. Elle provoque l'imaginaire, individuel et collectif, tout en sollicitant l'inconscient. Il en va de même du mot. Pour illustrer cela, j'aimerais citer ce que j'avais écrit pour la quatrième de couverture de 
Lecture d'une ville (1976) : "La ville, lieu privilégié de la rencontre, au croisement de l'imaginaire et du réel le plus contraignant, sans cesse l'homme d'aujourd'hui par elle se trouve requis. Mais le regard en impose aux lignes rigoureuses de la cité. Il la convie aux noces du désir, l'expose à des métamorphoses et, l'ajustant aux mots et aux phantasmes, l'intègre dans l'histoire secrète de chacun ".

H. B. : Bien sûr, l'amour est le ressort qui galvanise toutes les thématiques. Quand tu écris : "Chaque poème est la peau morte de ta mue ", il me semble que tu mets l'accent sur la métamorphose des sentiments. Est-ce que ce dynamisme émotionnel est à la source de l'amour et du lyrisme? Comment saccomplit son traitement poétique dans ton cuvre?

J.-M. T. : Une grande pudeur m'empêche de publier mes sentiments et, d'une façon générale, ce qui appartient à l'intimité. Rien d'autobiographique. Même si je dis " je ", il ne s'agit pas de mon vécu, ou alors tellement transformé qu'on n'en reconnaît rien. Cela va de pair avec ma réticence envers le lyrisme et mon choix d'une poésie de connaissance. Je ne vis évidemment pas sans amour. Il m'anime et me nourrit. En deçà de l'écriture matérialiste et objective, explosent en permanence les charges émotionnelles qui m'ébranlent. Je me compare volontiers à une caisse de résonance qui les amplifie au point de les rendre parfois insupportables. Cependant, on ne saurait confondre cet état avec la poésie qui vise l'universel et dans laquelle la gamme des émotions et des sentiments ne me paraît avoir d'intérêt que dans le dépassement de l'individuel et du subjectif. Je n'ai pratiquement jamais publié de poèmes d'amour, à l'exception de deux fois, à des moments d'intense passion, la première dans Mesure de la soif (1970), plaquette parue dans la collection " manuscrits " d'Encres Vives, la seconde dans la suite éponyme figurant au sommaire de L'oiseau de glaise (1995). Ce que tu nommes " métamorphose des sentiments " s'effectue conjointement avec d'autres métamorphoses philosophique, morale, politique, qui font que l'homme change à proportion de son expérience. Au fond, je me construis de tout ce qu’en moi je détruis. Le vers que tu cites prend en compte ce dynamisme émotionnel auquel tu attaches du prix, mais il met surtout en évidence les processus d'évolution de la poésie dans sa forme et dans son contenu. Muer, c'est changer de peau pour grandir et devenir autre. J'estime que tout moment de ma poésie doit être remis en cause et dépassé. Chaque phase contient des potentialités, des orientations, à exploiter. Il ne faut donc pas s'arrêter dans la relative satisfaction du niveau auquel on est parvenu et qui pourrait satisfaire 
quelqu'un dépourvu d'exigence. Le poème avance dans son reniement. Il faut apprendre à écrire contre soi-même, sachant que le terme du voyage n'existe pas et que l'important réside justement dans le voyage, le but dans l'aventure du langage chez le poète.

H. B. : J'ai écrit, dans le compte rendu d'un de tes livres, que ta "facture est d'une rigueur implacable. Aucune enflure de style, mais une maîtrise du langage... ». Es-tu d'accord avec cette évaluation? Si oui, élabore un peu. Si non, explique, sỉl te plaît.

J.-M. T. : Entièrement d'accord. Je te remercie de cette remarque pertinente. Je me garde de l'épanchement, des confidences, de l'exploitation un peu écœurante de l'intimité. Les maîtres mots de ma poétique sont, en effet, rigueur, exactitude, cohérence, maîtrise, lucidité, économie. Je m'y applique en toutes circonstances, conscient de ce que, pour être fiable, la poétique postule une éthique - et c'est à ce niveau, non à celui des circonstances, qu'on pourrait parler d'engagement. Je déteste le flou, le vague, l'à-peu-près, la complaisance, les facilités, le remplissage, le bavardage, l'emphase, etc. Ce sont des faiblesses humaines que leur manifestation en poésie amplifie. J'ai retenu les leçons de Roger Caillois dénonçant "Les impostures de la poésie ", dont les propositions ont fait grincer tant de dents gâtées. J'aime, au contraire, les œuvres qui me confortent dans ma propre recherche : la mathématique verbale de Perse, l'écriture aphoristique de Char, la méthode créatrice de Ponge, les figures relationnelles de Tortel, la tension radiale de Gaspar dans son approche de la parole. De mon point de vue, le poème doit s'imposer avec la force d'une évidence. Pour cela, il doit avoir un dessin net, suivre la courbe la plus adéquate à son objet, ne rien accepter que de nécessaire à sa fonction. L'écriture d'un poème demande un travail long et patient. L'intérêt réside dans cette difficulté qui consiste à dégager d'un premier jet, sorte de gangue, le poème potentiel. Nous sommes à l'opposé de la spontanéité. Le surgissement représente le premier état de ce qui deviendra, dans le meilleur des cas, poème. « À la levée du texte, la phrase s'ouvre raide et les mots collés, comme une paupière au réveil », dit Jean Tortel.

H. B. : Je crois que, dans toute ta poésie, on peut retracer un fil conducteur : celui du poète qui réfléchit à l'acte créateur. Est-ce vrai? Et pourquoi cette réflexion à l'intérieur même du corps du poème? 
J.-M. T. : La démarche créatrice inclut sa propre critique. Le poème est lieu d'interrogation sur lui-même. Il ne s'agit pas d'une critique discursive mais d'une réflexion organique. Le poème parle de la poésie en parlant d'autre chose, à commencer par le fait qu'à tout moment de son écriture se pose la question de savoir si les éléments utilisés relèvent ou non de la poésie, ou y contribuent. Quelquefois, c'est parfaitement délibéré, ça constitue même le sujet, par exemple dans ma plaquette Où sinvente le jour (2000), reprise dans Le Manteau de Circé (2003). La poésie se retourne sans cesse sur ellemême pour s'observer dans la distance et relancer son processus. Grâce à cette fonction la poétique s'affirme et évolue, dans une dialectique qui fonde la théorie et la pratique d'un même mouvement. L'important réside dans le fait que cette réflexion est spécifique, non généralisable. Ce qui vaut pour moi ne vaut pas pour un autre. Chaque poète l'exprime selon son propre usage. Pour reprendre différemment une de mes assertions, je dirais que les lois de fonctionnement d'un poème sont contemporaines de son inscription et ne valent que pour lui. À chaque poème, à chaque recueil, tout est à recommencer, sauf à se répéter, à se pasticher soi-même. Je crains, malgré la profonde admiration qu'il m'inspire, que, vers la fin de sa vie, René Char ne se soit laissé aller à faire du René Char, à tailler dans une langue morte dont il possédait sans effort la maîtrise. À ce point, on abandonne les ferments essentiels : l'incertitude, le risque.

H. B. : Peux-tu commenter les différents États des lieux de ta poésie? Et puis, peux-tu l'inscrire dans les mouvements actuels de la poésie française? Comment te démarques-tu par rapport à ces mouvements?

J.-M. T. : Je trace ma voie seul, sans m’occuper de savoir si ma poésie se situe dans un mouvement quelconque, si on la loue ici et si là on la blâme. Je vais plus loin, au risque de choquer, quand j'écris, que je suis pris par l'écriture, je ne pense pas à la façon dont les lecteurs — qu'ils soient ou non poètes - recevront mes textes. Ce n'est pas le moment. Qu'on ne dénonce pas en cela de l'orgueil. J'ai à résoudre un certain nombre de problèmes sur lesquels je concentre avec humilité toutes mes facultés. Il ne s'agit pas de prendre la pose, de cultiver le splendide isolement, de m'enfermer dans une tour d'ivoire, mais de répondre à une nécessité pratique imposée par ma conception de la poésie, ce qui postule une certaine disposition d'esprit. Le seul groupe auquel j’ai adhéré, et qui a exercé une influence déterminante sur mon travail de poète, est celui d'Encres Vives, à la grande époque : $1967-$ 1980 (j'appartiens toujours au comité de rédaction, même si les temps ont changé). Je me sens complètement étranger à des tendances comme la 
poésie du quotidien, de la banalité, parce que le travail sur la langue n'y est pas premier; à l'Oulipo, parce que les contraintes préexistent au texte et règlent sa production (cela me rappelle les jeux verbaux des Grands Rhétoriqueurs), tandis que je considère que chaque poème impose ses propres contraintes, qu'il faut reconnaître, et obéit à sa propre logique; aux performances, qui relèvent du spectacle plutôt que de la poésie, et ne peuvent se justifier de la provocation dès lors que plus rien ne choque une société blasée, où même les tabous sont devenus marchandises. Rien de cela ne m'intéresse. Un grand nombre de recueils me tombent des mains. J'y vois les manifestations d'une modernité factice, des conventions n'osant se présenter comme telles. Cependant, chacun a le droit de pousser sa chanson à sa guise. Si je ne m'inscris dans aucun mouvement cela ne signifie pas pour autant que je me situe hors de la poésie d'aujourd'hui. Je m'attache à des voix originales qui me touchent et me nourrissent. J'en ai déjà cité quelques-unes : Valéry, Perse, Caillois, Char, Ponge, Tortel. Parmi les poètes vivants, je retiendrais par exemple Yves Bonnefoy, Lorand Gaspar, Pierre Oster, pour rester dans le domaine français.

H. B. : " Dire le Sud, c'est le parler hors des langues battues. C'est en délimiter l'espace à l'intérieur de soi ". Je cite ce passage de Périmètre de l'arbre. J'aimerais que tu élabores, pour nous, l'aspect méditerranéen de ton écriture poétique.

J.-M. T. : Je suis un homme du Sud, un Méditerranéen. Je refuse cependant de tomber dans le travers réducteur du régionalisme, tout en reconnaissant mon attachement à Marseille, ma ville natale, et à la Provence. J'ai écrit : «Ma terre, je la porte à l'infini ». Par la branche maternelle, je suis lointain petit cousin de Frédéric Mistral. Cette filiation n'est pas indifférente même à considérer que, poétiquement, je me situe à l'opposé de l'auteur de Mireille. Je ne parle ni ne comprends le provençal, sans regret. Je suis un Provençal d'expression française. Si le hasard m'avait fait naître à Strasbourg, à Lille ou à Rennes, j'aurais sans doute été poète, mais pas le même poète. Le climat dans lequel j'ai été élevé, l'environnement, ont contribué à la formation de ma sensibilité. Ils ont révélé ou accusé des tendances de mon caractère. Le Sud oblige au face à face avec la lumière qui taille un relief aux traits nets. La réflexion a sa place, pas l'imagination, l'énigme, non le mystère. Ce qui se donne à voir se donne à lire, à déchiffrer. On ne ruse pas avec l'évidence. On ne ment pas sur les distances. On entre dans une géométrie sensible, une ivresse lucide. Tous les masques tombent sous le regard de Zeus. Le langage 
radiographié laisse voir ses os, comme la terre ses tendons. Écoutons Pierre Toreilles qui m'est proche : "La lumière pour nous/découpe l'espace/et donne enfin au jour/l'abondance qui lui manquait ». Or la lumière acquiert ici un caractère spécifique en ce que son lieu d'origine change, se renverse la source jaillissante ou immanente. Elle devient propriété de la matière comme le saisit et le rend le pinceau de Cézanne. La montagne et le mot sont des blocs de lumière. L'écriture poétique cherche à la libérer, et de soimême. On ne saurait prétendre dire le Sud sans en écarter la poussière, trouer le décor, arracher les langues mortes, les contresens sur sa splendeur. L'écriture méditerranéenne se reconnaît à son économie. Non sans une certaine dureté, elle taille dans le langage des équivalences minérales.

H. B. : En essayant d'articuler l'eternel en l'Homme, tu convoques le mythe et la mémoire. Surtout dans les réalités précises du langage. Comme dans ton recueil Chasseur de mémoire. Explicite et commente un peu ces remarques.

J.-M. T. : Le groupe Encres Vives a beaucoup travaillé sur le mythe, le pouvoir créateur de l'imaginaire dans sa relation au réel. Sans préjudices de ceux qui furent fondateurs, nous vivons au milieu des mythes, nous les engendrons à partir de notre propre substance. Ils surgissent au terme de la traversée du langage. J'ai consacré autrefois une étude à ce phénomène que j'ai baptisé mythécriture dans mon essai Vers une logique poétique (1980). Il s'agit pour moi de consentir aux sacrifices nécessaires pour accéder à l'universel. Non pas les mesquineries qui affectent l'individu, ses petits secrets, ses intérêts minables, mais une visée plus haute tendue vers une certaine grandeur qui fonde l'Homme et permet à chacun, selon son destin, de se reconnaître en lui. L'universel ne manifeste pas la fuite du réel mais un effort pour l'englober tout entier et lui donner un sens partageable. Sur ce point, j'aime à citer Valéry : "Quiconque n'a pas tenté de se rendre semblable aux dieux, c'est moins qu'un homme ». Je ne suis pas tourné vers le passé. Je ne reviens pas volontiers sur mes pas. Je n'éprouve ni mélancolie ni nostalgie. M'attire l'inaccompli, la zone d'ombre qui me précède. Cependant, je suis porté par ce qui fut. Je vis de ce que j'ai brûlé. Pas seulement moi, mais l'histoire. Nous marchons sur des morts. Le problème de la mémoire m'apparait donc essentiel. Dans Chasseur de mémoire (2001), j'ai tenté de conjuguer, en exploitant les ressources du langage, la mémoire individuelle, la mémoire collective, celle qui est inscrite dans la terre, la mémoire des mots, la mémoire cosmique, pour parvenir à ce que Jean Orizet appelle " un chant orphique général ». Une phrase du "Silence des dieux» résume ma poétique - lyrisme des profondeurs, la relation avec 
la science, la corrélation des ordres — : " Précaire, ô fugace, devenir le chant que je suis! Relier d'une même vibration, sur l'invisible corde qui me tend, la plus infime unité de mon être à l'amorce d'une pensée ».

H. B. : Je reviens à ta poésie en prose, donc un peu en aval de ton itinéraire. Ce sont presque toujours de petits fragments repliés sur eux-mêmes. Des éclats, pour ne pas dire des éclairs de pensée. Pourquoi avoir recours à ce pracédé? Quels en sont les avantages? Les inconvénients?

J.-M. T. : Le problème ne se pose pas en termes d'avantages et d'inconvénients. J'ai proposé plus haut une analyse élémentaire du poème en prose et justifié mon choix. Dans certains cas, en fonction de la quête de ce qu'il y a à dire, le poème en prose offre la réponse la plus adéquate. À d'autres, c'est le poème en vers libre, la laisse, le verset. Rien d'arbitraire en cela, mais une conscience aiguë des conditions et des enjeux. La distribution par fragments me vient naturellement, sans doute parce qu'elle correspond à une réalité profonde. Je tiens ici pour secondaire la diffusion et le rôle du fragment dans la littérature contemporaine. Considérons toutefois que le fragment postule l'existence d'un ensemble, supposé ou réel, duquel il se détache. Mes proses constituent, au contraire des unités denses et compactes, qui pourraient se suffirent à elles-mêmes. Elles sont comme des coups de sonde successifs lancés dans les ténèbres de l'inconnu d'où elles sont supposées extraire un peu de lumière noire. Leur nombre circonscrit l'espace de cette opération. En conséquence, il existe une unité thématique mais pas d'unité organique. Je procède plutôt par juxtaposition. J'accepte ton interprétation d'éclats ou d'éclairs de pensée puisqu'en peu de mots, peu de lignes, il est possible de lier la fulgurance et l'intensité. On n'habite pas l'éclair en permanence. La trame textuelle du poème en prose, en ce qu'il rassemble tout en son lieu, permet de mieux le capter et le conserver. Cependant, on peut aborder le sujet sous un autre angle. Réflexion poétique, réflexion philosophique, ne cessent de s'entrelacer, et forment une tresse abstraite, quoique sensuelle et sensible. Elle se développe selon un flux qui, à certains moments, enfle brusquement sous la poussée du sens, un peu comme dans une courbe on distingue les nœuds et les vents. Le besoin d'écrire naît à ce moment-là, quand l'activité cérébrale s'accélère et atteint un niveau plus élevé. La continuité du poème en prose permet l'enregistrement et la conservation de ces moments exceptionnels. Ce ne serait pas le cas avec le découpage des vers et l'usage du blanc. 
H. B. : Au fond, ces paragraphes donnent l'allure d'une prose poétique descriptive qui privilégie, du moins à la surface, l'impersonnel, l'objectif, le narratif. Quelles sont les raisons de ce choix délibéré?

J.-M. T. : Oui, une prose poétique descriptive du phénomène poétique qui ne peut atteindre son objet que par l'éloignement du subjectif au profit de l'impersonnel. J'évite le plus souvent les pronoms personnels. J'utilise de préférence l'indéfini ou pas de pronoms du tout. Décrire, en l'occurrence, revient à s'appliquer à une réalité intérieure, à en matérialiser l'existence dans ses différents aspects en posant au plus juste les mots appropriés. Une description des phénomènes plutôt que la description statique, premier état de toute science, comme la botanique ou l'anatomie. Cela m'empêche d'aller dans tous les sens selon mon humeur ou les stimulations de hasard. Je me réfere sans cesse à cette rigueur. Quand j'écris : "L'éclair d'un chant perdu lézarde la mémoire ", c'est de cela qu'il s'agit. D'où la recherche de la formule qui colle le mieux, la courbe la plus tendue, sans un mot de trop ni qui ne soit à sa place. Dans cet esprit, je respecte l'ordre de l'énonciation. Les antépositions, qui colorent d'affectivité, sont assez rares dans mes poèmes. De même les adjectifs, que je n'utilise qu'à dessein, la rareté conférant alors plus de force. Par exemple : "De frêles boules de fibres traversent la mémoire ". Jamais d'effets de style sinon malgré moi, faute de vigilance. En 1979, un critique voyait dans ma poésie un "vertige de l'impersonnalité ». Ce n'est pas exact. D'abord, parce que la personnalité se manifeste aussi et surtout par une vision du monde, un univers intérieur, et par la spécificité des moyens mis en ouvre pour les communiquer - ce fameux style qui fait l'homme. Ensuite, parce que les émotions, les sentiments, ne sont point absents. Simplement, ils ne sont pas où on s'attend à les trouver. Ce sont eux qui produisent la tension sous l'écorce, sous la peau des mots. Ils ne sont pas exprimés en clair, ils émanent de la chaîne verbale, et contribuent à ce climat dont nous avons traité. J'ajoute que cela ne traduit pas seulement une pudeur excessive. Que doit la beauté scintillante d'une comète à l'émotion du spectateur? Quand j'écris, dans États du lieu, " De grands pans de murs disent encore ce que fut la demeure. Et que chacun la crut solide ", il s'agit de moi parvenu au terme de la maturité. On l'aura compris, je parle d'un lieu d'origine qui est celui du créateur ou, si l'on préfere, du producteur de textes. Je n'aborde pas ce qui concerne le récepteur, le lecteur, à qui je n'ai rien à imposer.

H. B. : Dans Le Manteau du Circé, tu écris : "Le poète pris dans les roseaux du songe ne connaît pas sa perte ». Pourquoi et d'où vient cette perte? Surtout 
quand tu conclus ce poème par "Le silence sera ta couche ". Et, dans un autre, "Prends garde au partage des mots". Peux-tu développer cette ambigü̈té fondamentale entre parole et silence? N'est-elle pas source de poésie?

J.-M. T. : La poésie est une aventure, une sorte d'Odyssée dans l'océan du langage, dont on ignore où et quand elle débouchera. Le mot songe, dans ta citation, ne désigne pas seulement l'état de rêve éveillé, mais, selon l'acception ancienne, un labeur long et pénible, par exemple dans ce vers de Clément Marot, dans la fable du lion et du rat, quand ce dernier ronge les mailles du filet pour délivrer le roi des animaux : "Vrai est qu'il y songea assez longtemps ». De même l'emploi du verbe connaître est à prendre de deux façons : n'a pas conscience de sa perte; et n'arrive jamais à se perdre, ne connaît pas ses limites. Pardonne-moi de faire un peu d'explication de texte. Le roseau - plante symbolique de l'homme - a été utilisé pour écrire (le calame), et il existe, surplombant le monde, sur les hauts plateaux andins où réalité et songe se confondent, une civilisation du roseau. L'idée directrice ne vaut pas pour ce seul poème. Le poète, dans sa progression, s'enrichit et se conforte de tout ce qu'il perd. Nous avons déjà abordé le problème de la mue qui rejoint celui-ci. Le poème, en son surgissement, est une déchirure du silence (mon ami Jacques Lovichi a d'ailleurs intitulé un de ses recueils Fracture du silence). Nous écrivons pour donner au silence une forme habitable (Yves Broussard titre un recueil de 1988 : Paroles du silence). Ces remarques prouvent combien cette question préoccupait les poètes de la revue Sud, dont je faisais partie. Quand nous aboutirons au silence éternel, à l'instant de la perte définitive, ce sera comme si nous ne l'avions jamais troublé de nos mots. Dans "Bestiaire du silence ", texte inaugural de L'Instant précaire (1995), j'écris : "Le silence de la page est peuplé. Il s'ouvre sur des présences incertaines ». Le choc du silence et de la parole (j'entends la parole vraie) est semblable à celui du silex. Il engendre l'étincelle qui a nom poésie.

H. B. : Jean-Max, y aurait-il une question occultée, à laquelle je n'ai pas pensé? Tu aurais voulu que je te la pose? Eh bien, pose-la. Et réponds-y.

J.-M. T. : Ton questionnaire porte principalement sur la poésie, qui m'importe le plus. Nous n'avons pas parlé du roman, auquel je suis venu tard, et un peu par défaut. Je voulais exprimer ma révolte contre la violence de notre époque, son cortège d'horreurs. Les récentes guerres des Balkans ont servi de déclencheur. Or, l'espace du poème, tel que je le conçois, ne le permettait pas. J'avais besoin de l'espace géographique, de personnages, 
d'action. J'ai donc écrit Le Jardin d'argile (1997). Je me suis alors avisé des diverses présences et fonctions possibles de la poésie dans le roman, à commencer par l'utilisation de grandes métaphores génétiques. Je m'en suis servi dans mon dernier roman, L'Homme chargé d'octobres (2005). Et c'est encore le cas dans Le Capitaine d'armada, celui auquel je travaille. Bien sûr, cette articulation des deux formes d'écriture, poétique et romanesque, mériterait plus ample développement. Mais ce n'est pas le lieu.

H. B. : J'aimerais te remercier d'avoir répondu si gracieusement à mes questions. J'espère que ce tour d'horizon a plus ou moins couvert l'ensemble de ton ceuvre. J'espère aussi que cet entretien suscitera l'envie de lectures sans cesse renouvelées de tous tes livres. Je suis convaincu que tes lecteurs ne seront pas déçus.

\section{Bibliographie de Jean-Max Tixier}

\section{Principales ceuvres poÉtiques}

La Poussée des choses (prix Encres Vives, éd. Encres Vives, 1967), La Pierre hypnotisée (prix Blaise Cendrars, éd. Nouveaux cahiers de Jeunesse, 1968), Lecture d'une ville (éd. Sud, 1976), La Traversée des eaux (éd. Sud, 1984), L'Arrière-Temps (éd. La Table Rase/Le Noroît, 1989), États du lieu (prix Campion-Guillaumet de la Société des Gens de Lettres, prix Louis Guillaume, éd. Autres Temps, 1989), L'Instant précaire (éd. L'arbre à paroles, 1995), L'Oiseau de glaise (prix Antonin Artaud, éd. Arcantère, 1995), Chasseur de mémoire (éd. Le Cherche Midi, 2000), Le Manteau de Circé (bourse Thyde Monnier de la Société des Gens de Lettres, éd. Le Taillis pré, 2003).

\section{ROMANS}

Le Jardin d'argile (prix Antigone, éd. Le Cherche Midi, 1997), Le Crime des Hautes Terres (éd. Les Presses de la Cité, 2001), La Fiancée du santonnier (éd. Les Presses de la Cité, 2002), Le Maître des roseaux (éd. Les Presses de la Cité, 2003), Marion des Salins (éd. Les Presses de la Cité, 2005), L'Homme chargé d'octobres (éd. Le Cherche Midi, 2005).

\section{EsSAI}

Vers une logique poétique (éd. La Table Rase, 1980) 